\title{
Current role of imaging in COVID-19 infection with recent recommendations of point of care ultrasound in the contagion: a narrative review
}

\author{
Darshan Gandhi ${ }^{1} \wedge$, Nitin Jain ${ }^{2}$, Kanika Khanna ${ }^{3}$, Shuo Li $^{4}$, Love Patel ${ }^{5}$, Nishant Gupta ${ }^{6}$ \\ ${ }^{1}$ Department of Radiology, Northwestern Memorial Hospital, Northwestern University Feinberg School of Medicine, Chicago, IL, USA; \\ ${ }^{2}$ Department of Radiology, Ascension St. John Macomb and Oakland Hospitals, Warren \& Madison Heights campuses, Troy, MI, USA; ${ }^{3}$ Department \\ of Radiology, Abdominal Imaging, Wayne State University School of Medicine, Henry Ford Hospital, Detroit, MI, USA; ${ }^{4}$ Department of Radiology, \\ KU School of Medicine-Wichita, University in Wichita, Wichita, KS, USA; ${ }^{5}$ Department of Internal Medicine, Abbott Northwestern Hospital, \\ Minneapolis, MN, USA; ${ }^{6}$ Department of Radiology, Bassett Healthcare, Cooperstown, NY, USA \\ Contributions: (I) Conception and design: D Gandhi; (II) Administrative support: D Gandhi, N Jain; (III) Provision of study materials or patients: \\ K Khanna, S Li; (IV) Collection and assembly of data: L Patel, N Gupta; (V) Data analysis and interpretation: None; (VI) Manuscript writing: All \\ authors; (VII) Final approval of manuscript: All authors. \\ Correspondence to: Darshan Gandhi, MD. Department of Radiology, Northwestern Memorial Hospital, Northwestern University Feinberg School of \\ Medicine, 676 N St. Clair St, Suite 800, Chicago, IL 60611, USA. Email: darshangandhi7@gmail.com.
}

\begin{abstract}
Radiological studies have an important role in the diagnosis and follow up of many infectious diseases. With current pandemic of Coronavirus disease 2019 (COVID-19) though the molecular analysis with reverse transcriptase polymerase chain reaction (RT-PCR) remains the cornerstone of diagnosis, the critical role of chest imaging including CT scan and baseline X-ray became apparent early in the course due to concern for less than optimal sensitivity of PCR testing. Delay in molecular diagnosis due to a shortage of testing kits and laboratory personnel also makes imaging an important modality in early diagnosis for appropriate triage and isolation decisions. CT scan technology is widely available in developed parts of the world but in developing countries, CT scanner is not widely available especially in rural settings. CT imaging usually requires patient movement to the radiology department and the scanner is not easy to disinfect. Point of care ultrasound (POCUS) has been used for many years in the assessment of critically ill patients in emergency departments and intensive care units. It is rapidly gaining popularity across many specialties and part of many general medicine training programs across the United States. It can be learned rapidly and with experienced hands, POCUS can help identify disease patterns in the lung parenchyma, and during the current pandemic has been gaining special attention. In this article, we review the most prominent imaging findings on chest X-ray and CT scan in patients with COVID-19. We also focus on the background and evolution of POCUS with studies showing the promising role of this diagnostic modality in COVID-19 infection. In addition, we describe step by step guidance on the use and disinfection of the portable ultrasound machine.
\end{abstract}

Keywords: COVID-19; coronavirus; Wuhan; point of care ultrasound (POCUS); crazy-paving; CT; reverse transcriptase polymerase chain reaction (RT-PCR)

Submitted Apr 01, 2020. Accepted for publication Jul 31, 2020.

doi: 10.21037/atm-20-3043

View this article at: http://dx.doi.org/10.21037/atm-20-3043

^ ORCID: 0000-0002-7079-450X. 


\section{Introduction}

Radiological exams are of high value in the detection, management and follow up of Coronavirus Disease 2019 (COVID-19) infection cause by Severe Acute Respiratory Syndrome Coronavirus 2 (SARS-CoV-2). After first cases reported in Wuhan province of China in later 2019 at the time of this writing the pandemic has involved over 8 million people across 188 countries (1). Though current gold standard for diagnosis of COVID-19 is clinical presentation with confirming viral presence with positive reverse transcriptase polymerase chain reaction (RTPCR), barriers including testing kit availability and concern for sensitivity have been raised. In a study of more than 1000 patients in Tongji Hospital in Wuhan, China, it was concluded that computed tomography (CT) can be used as a first line screening tool for COVID-19. The sensitivity of CT imaging findings in COVID-19 has been shown to be as high as $98 \%$, compared to RT-PCR sensitivity of $71 \%$ (2). Therefore, patients with COVID-19 illness can demonstrate important findings on CT with an initially negative RT-PCR (3). Due to overwhelming number of patients getting infected with COVID-19 infection role of ultrasound in management of COVID-19 patients should be discussed. A recent study involving point of care ultrasound (POCUS) showed strong correlation between bedside US in emergency department and CT findings of lung in COVID-19 patients in Italy (4). Though lung is a primary organ involved in COVID-19, the disease can impact other organs either from direct viral involvement or injury from inflammatory reaction. It has shown to affect heart with myocarditis, pericarditis, stress cardiomyopathy and arrhythmia $(5,6)$. POCUS also provides advantage in assessment of heart and lung at the same time. It is also feasible to use it as a handy tool especially in developing part of world due to its portability and ease of disinfection of ultrasound probe and machine in comparison to CT scanner room. In following review, we discuss imaging findings of COVID-19 in chest radiography, chest $\mathrm{CT}$ and rapidly evolving role of POCUS. We present the following article in accordance with the Narrative Review reporting checklist (available at http://dx.doi.org/10.21037/atm-20-3043).

\section{Imaging findings of COVID-19 in chest radiography}

Chest radiographs have limited sensitivity for early disease and can be normal even when ground glass opacities are seen on chest CT performed at the same time. Therefore, it is not recommended as the first line imaging test. In later disease or severe infection, radiographs can show bilateral multifocal consolidations and can even present as a "white lung" with small effusions (Figure 1) (7). In addition, nonspecific imaging findings such as hazy infiltrates make it difficult to arrive at a more definitive diagnosis, as opposed to CT where findings are more unique and better described (3).

\section{Imaging findings of COVID-19 on CT scan}

CT is a more effective test for early detection of COVID-19 pneumonia. CT is easy to perform, fast, readily available and has a high sensitivity. It can provide valuable information for further diagnosis as well as prevention and control of COVID-19. The recommended protocol for chest CT in COVID-19 is to obtain images at 1-mm thick contiguous slices, with reformatted lung and soft tissue windows. Intravenous contrast is not essential.

The typical findings on chest CT are multifocal ground glass opacities (GGO, defined as hazy opacity that does not obscure the underlying pulmonary vessels and bronchi) in a subpleural distribution in bilateral lungs with occasional patchy consolidation (defined as airspace opacity that obscures underlying parenchymal vessels and bronchi). This is the hallmark finding described across several studies (Figures 2,3) (8). Posterior and lower lobe predilection has been observed (3). It is important to note, however, that these appearances are not unique to COVID-19, as GGO can be seen in both other types of alveolar and interstitial processes. In later stages of this disease, crazy paving pattern is often seen which is described as GGO with superimposed inter- and intralobular septal thickening. Pure consolidation is less common. Reverse CT Halo signs can be seen (GGO surrounded by a halo of consolidation). Pleural effusions and lymphadenopathy are atypical or uncommon findings and in one of the early studies, $\mathrm{Ng}$ et al. mentioned that these findings are not seen in their 21 COVID-19 cases from Shenzhen and Hong Kong (9). In another one of the early studies done at the epicenter Wuhan, China, they mentioned that pleural effusion was seen in around $9 \%$ of patients from total of 62 patients and in another large systemic review of 919 patients, only $7 \%$ of patients showed atypical findings like pleural effusions, lymphadenopathy, bronchial wall thickening, and parenchymal lung nodules surrounded by GGO $(10,11)$. Multiple tiny nodules 

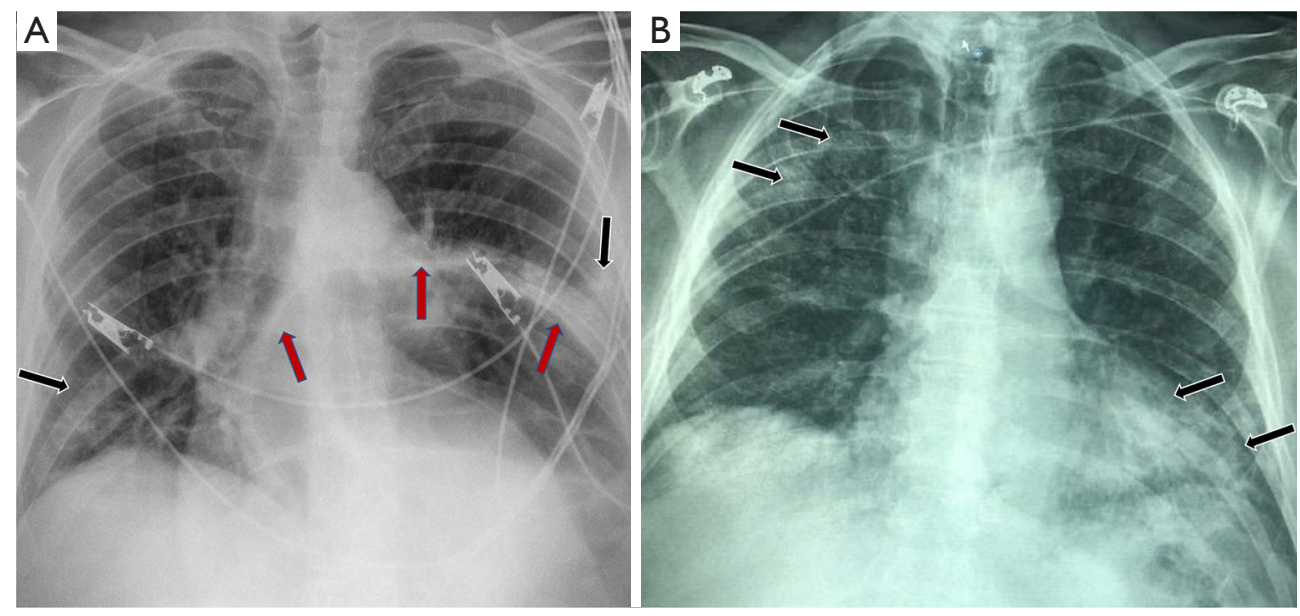

Figure 1 (A) A 66 years old male came with cough, shortness of breath in emergency department shows hazy opacities in both lung bases (black arrows) who later turned out to be positive for COVID-19 [also see large hiatal hernia in retrocardiac region (red arrows)]. (B) Another 63 years old male with COVID-19 infection shows multifocal bilateral air-space opacities (black arrows) in both lungs.
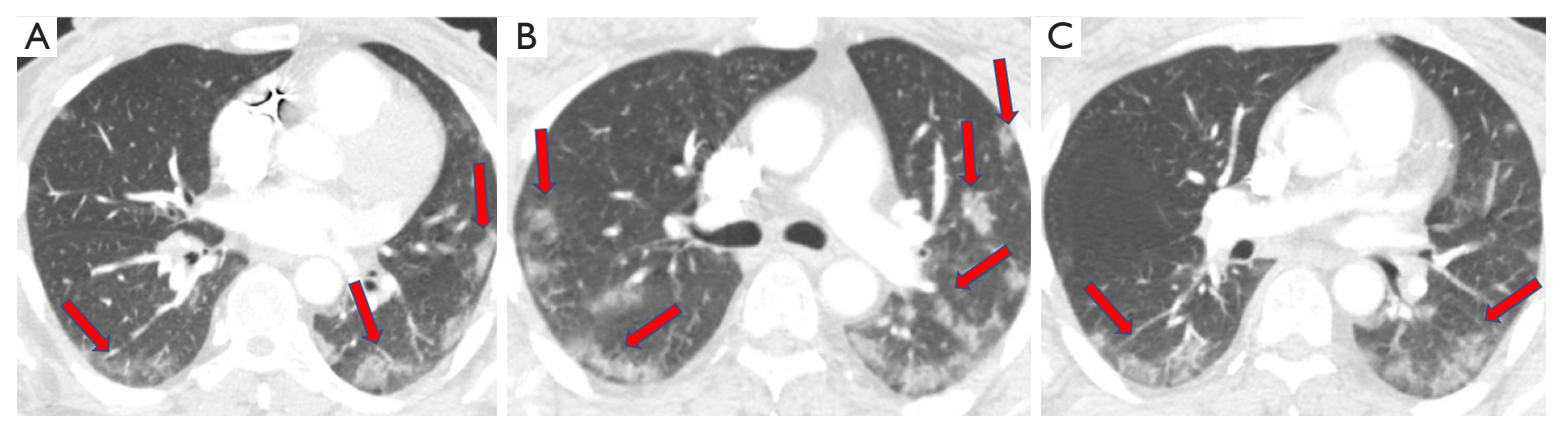

Figure 2 A 57 years old male who is known case of Brugada syndrome came with fever, sort throat and cough show bilateral predominant consolidative opacities with subtle ground glass opacities in striking peripheral distribution (red arrows) in both lungs in multiple axial images (A,B,C).
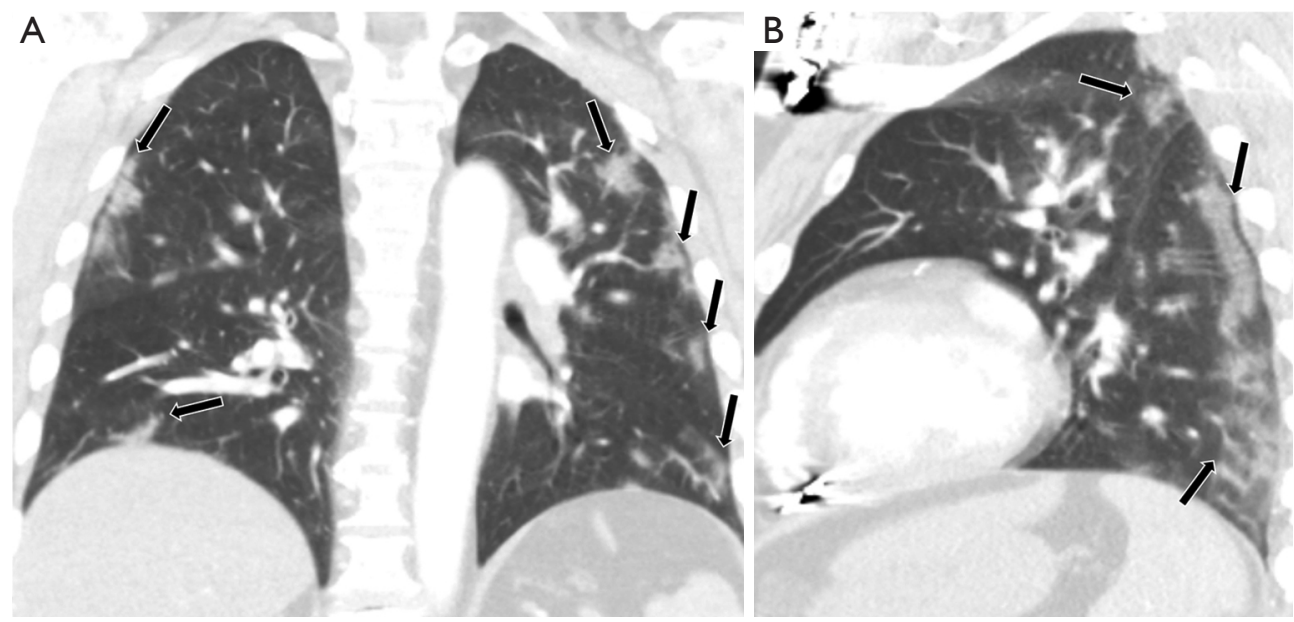

Figure 3 Coronal (A) and sagittal (B) views of the patient shown in Figure 2 again showing bilateral consolidative with subtle ground glass opacities in peripheral distribution (black arrows). 
and cavitation are rare and should raise the possibility of bacterial superinfection or alternative diagnosis (9). Centrilobular ground glass nodules have been described in a study by Liu $e t$ al. where they studied a familial cluster of cases of COVID-19 infection (12). Reports have also described an organizing pneumonia pattern with multifocal nodular opacities in multiple lobes (13).

An asymptomatic patient with chest CT abnormality showing consolidation has been reported in a study by $\mathrm{Ng}$ et al. (9). Follow-up CT in this patient with a history of travel and positive contact showed improvement in the CT findings 4 days later toward normal lung findings.

It should be noted that up to $50 \%$ of patients with RTPCR confirmed COVID-19 infection may have normal chest CT findings with early symptom onset and a negative CT scan should not exclude the diagnosis of COVID-19 in a patient with clinical symptoms and travel or exposure history $(3,14)$. This suggests that imaging findings may not correlate with disease symptoms.

Besides aiding in the diagnosis of COVID-19, CT can be useful for assessing the severity of the disease which further helps guide clinical management. In severe infection, CT shows diffuse consolidation with air bronchograms and bronchiectasis, also called as "white lung" when most of the lungs are involved. In mild infection or non-ICU patients, CT shows bilateral GGO and subsegmental consolidations (Figures 2,3) (7).

Another advantage of CT is its utility in categorizing imaging patterns based on the time course of disease. In a study by Pan et al., who reviewed 121 CT of COVID-19 positive patients at 4 -day intervals, it was seen that frequency of CT findings is related to the time course of the infection (15). Four patients showed negative CTs without any findings in $0-2$ days after symptom onset, however, repeat CT imaging in these patients showed positive lung findings. This reinstates the fact that in early disease, chest CT has limited sensitivity and negative predictive value, and should not be used as a standalone tool to rule out COVID-19 infection.

While GGO is seen in early infection, findings of consolidations, total lung disease, bilateral involvement, crazy paving appearance, linear opacities and peripheral distribution are thought to represent the organizing phase of the disease and are more frequently seen in patients who have had symptoms for a longer time.

Temporal changes in follow-up CTs over time in COVID positive patients are helpful in predicting disease progression, recovery and development of complications as they reflect typical viral induced lung injury. After symptoms begin, the degree of CT findings progresses rapidly, peak at days 6-11 of illness and then show persistent high levels of abnormalities demonstrating slower resolution (16).

The primary pattern of abnormality after symptom onset (days 0-5) was ground-glass opacity, out of which pure GGO is the most common subtype and GGO with superimposed inter and intralobular lines (crazy-paving) was seen to be second most common subtype of GGO (Figure 4). GGO with irregular linear opacities and interfaces is the other common subtype of GGO which was seen to peak on days $6-11$. Between days $0-11$, consolidation was the second most common pattern seen on CTs. Mixed patterns were seen to peak on illness days 12-17. Most of the patients discharged showed residual disease on CT with GGO being the most common appearance. It is interesting to note that only pure ground-glass opacity could be observed in CT scans of otherwise healthy patients, prior to the onset of symptoms $(15,16)$. Another interesting observation was that pure GGO was seen to have a declining then rising trend with a second peak seen at days 18-23 with the other patterns slowly decreasing. This suggests that groundglass opacity during the final stage of the infection likely represent absorption of the illness (16). Pan et al. concluded COVID-19 CT-imaging findings in 4 stages based on the number of days from symptom onset (15):

(I) Early stage (0-4 days)-GGOs in subpleural lower lobes of lungs;

(II) Progressive stage (5-8 days) - multiple, bilateral affected lobes with diffuse GGOs, crazy-paving and/or consolidation;

(III) Peak stage (9-13 days)—extensive GGOs, dense consolidation, crazy-paving pattern, with or without residual parenchymal bands;

(IV) Absorption stage (14 days or more)-gradual absence of crazy-paving pattern and resolution of consolidation, GGOs.

Another potential clinical application of CT in followup of COVID-19 patients is assessment of inflammatory burden by using volume rendered CT (17). By 3D volumetric render of the lungs, this allows for a quantitative assessment of the degree of which the lungs are involved.

\section{Imaging findings of COVID-19 with POCUS}

POCUS is a safe and rapidly evolving diagnostic modality in current health care. Traditionally, ultrasonography has been used by imaging specialists, such as radiologists 

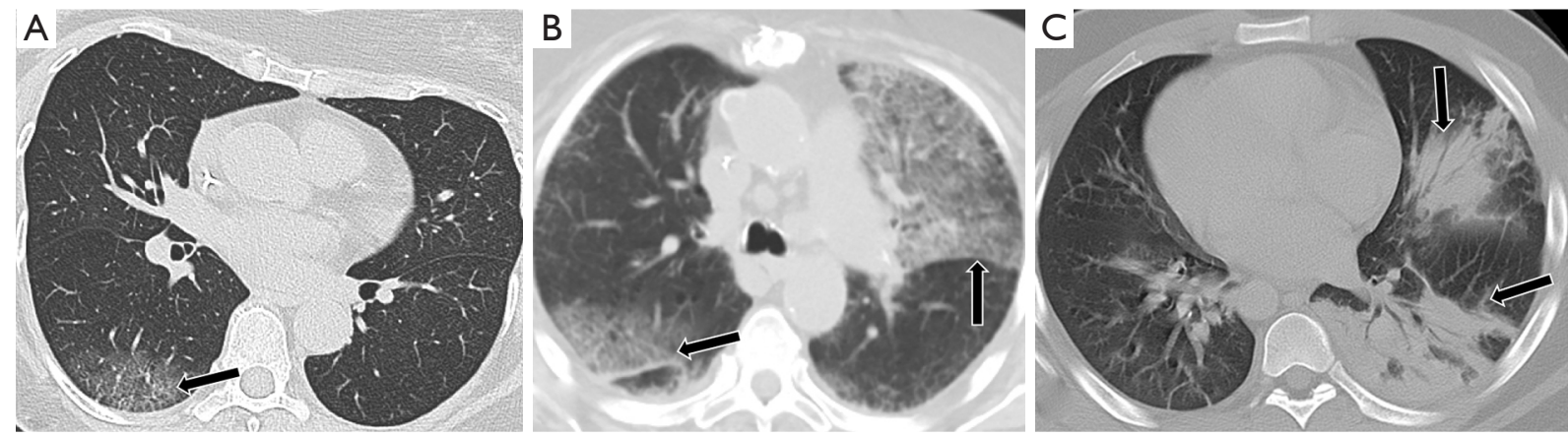

Figure 4 Few more patterns of CT images of COVID-19 patients with RT-PCR positive results. A 67-year-old patient with cough and dyspnoea shows focal ground glass opacification with crazy-paving pattern (black arrow) in right lower lobe (A). Another 80-year-old patient with known cardiac disease showing diffuse ground glass opacification with crazy-paving pattern (black arrows) in lingula and right lower lobes (B). A 67-year-old patient shows multifocal subsegmental consolidative opacities (black arrows) in lingula and left lower lobe (C).

and cardiologists; however, it is now utilized by health care professionals from nearly all specialties (18). Ultrasonography do not generate ionizing radiation and can be used to evaluate the lungs for detection of pneumonia at bedside. Since beginning of COVID-19 pandemic discussion about role of POCUS has intensified due to added risk of infection transmission with patient transportation to imaging areas.

Historically use of ultrasound in evaluation of pleura and lung parenchyma was thought to limited in radiology as healthy lungs back reflects ultrasound waves leading to horizontal reverberation artifacts. In a landmark study in 1987, Wernecke et al. described ultrasonographic appearance of pleura, pleural movements against lung parenchyma and role in evaluation of pneumothorax (19). Since then thoracic ultrasound has slowly gained more popularity to a point where a metaanalysis in 2011 showed in experienced hands ultrasound has higher sensitivity and similar specificity to X-ray in detection of pneumothorax (20).

Ultrasound waves reflected back by healthy lungs are called A lines which are transverse parallel hyperechoic lines. As the lungs are affected by disease process variety of ultrasound pattern emerge including $\mathrm{B}$ lines or so called "lung rockets" which are vertical hyperechoic lines which starts from pleura and travel to the bottom of the image and seen in edema or fibrosis of interlobular septa $(21,22)$. In pneumonia increased echogenicity of affected lungs appears similar to liver with presence of air-filled bronchi seen on both static and dynamic images also called as "tissue pattern" $(22,23)$. Absence of both A lines and $\mathrm{B}$ lines are additional criteria to diagnose consolidation or pneumonia (22). The most specific sign of pneumonia on chest ultrasound is the "Shred Sign", which is the irregular, "shredded" appearance seen at the interface between aerated normal and consolidated abnormal lung (Figure 5) (24).

With description of these patterns, lung ultrasound in evaluation of respiratory failure and management of critically ill patients is well studied and used widely $(25,26)$. Lung ultrasonography gives the results that are similar to chest CT and superior to standard chest radiography for evaluation of pneumonia and or adult respiratory distress syndrome (ARDS) with the added advantage of ease of use at point of care, repeatability, absence of radiation exposure, and low cost $(27,28)$.

In a study published in 2017 in the European Respiratory journal that was performed on 55 patients, chest ultrasound was seen to have $100 \%$ sensitivity, $91 \%$ specificity and $98.2 \%$ accuracy in the diagnosis of pneumonia in comparison to chest radiographs which had a sensitivity of $74.4 \%$, specificity of $25 \%$ and accuracy of $63.3 \%$. Another study by Long et al. which was a meta-analysis of 12 studies, including 1,515 adult subjects concluded that lung ultrasound is helpful to diagnose lung pneumonia with high accuracy $(29,30)$.

Recent study involving critically ill patients with acute respiratory failure 9 areas protocol lung ultrasounds not only outperformed portable chest X-ray but also had lobe specific correlation with CT chest findings (31). Lung ultrasound is more useful when findings are located on peripheral part of lungs.

Few small studies have described lung ultrasound findings in COVID-19. Although this modality cannot 

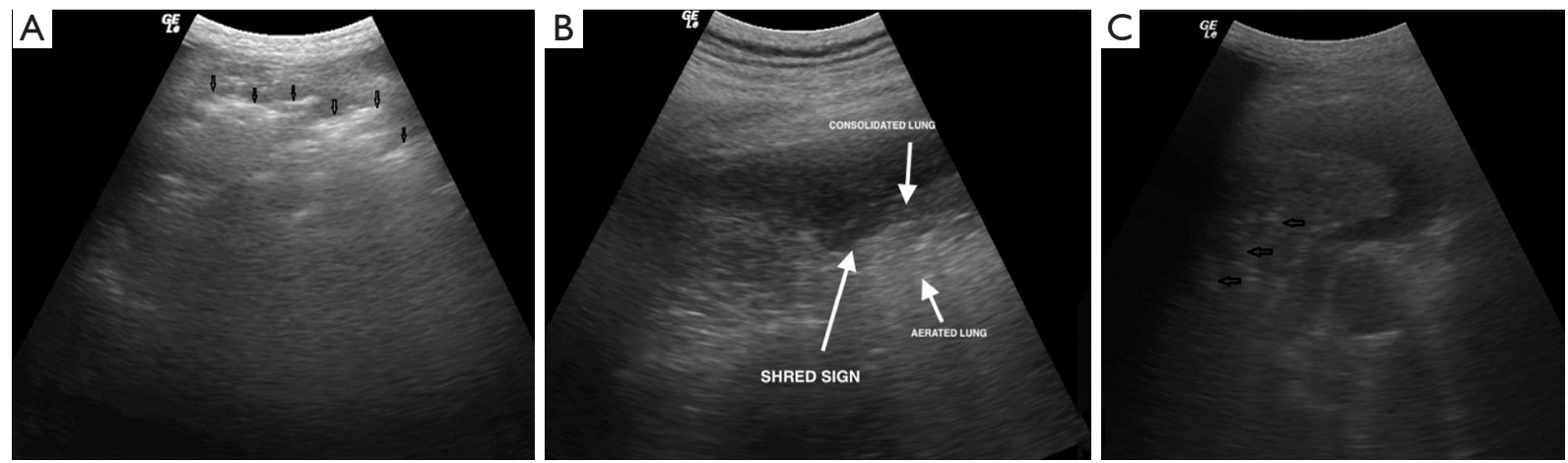

Figure 5 US imaging of the right lower chest shows shredded appearance between normal aerated lung and consolidated lung, also known as "shred sign" seen with arrows in (A) and (B). (C) More linear echogenicity in consolidated hypoechoic lung tissue showing hepatization, also called air bronchograms (black arrows).

differentiate between bacterial and viral pneumonia, it is a valid and easy technique, without use of big equipment or machinery. Characteristic findings described in one study were thickening of the pleural line with pleural line irregularity, B lines in a variety of patterns including focal, multifocal, and confluent. Consolidations in a variety of patterns including multifocal small, nontrans lobar, and trans lobar with occasional mobile air bronchograms and appearance of A lines during recovery phase. Pleural effusions were uncommon (32). In another study with 20 patients, the lesions are mainly characterized by a large number of B lines, sub pleural pulmonary consolidation and poor blood flow on doppler in areas with consolidation (33). Of note a small study by investigators in Italy found correlation of CT findings with lung US in COVID-19 infection (4).

In a recent review article compiling current available literature of lung ultrasound use in COVID-19 suggested lung ultrasound can help define severity and progression of COVID-19. Mild to moderate disease will show scattered $\mathrm{B}$ lines, irregular and thickened pleural line, areas of $\mathrm{B}$ lines alternating with normal lung sliding and A lines called "skipped lesions" and small consolidation $(\sim 1 \mathrm{~cm})$. In severe disease B lines become confluent and size of consolidation increases. In critical COVID-19 patient shows ultrasound findings of extensive coalescent and disappearing B lines affecting upper and anterior areas of lungs, significant small consolidation of upper and anterior areas of lungs and bilateral interstitial pattern with consolidation with or without air bronchograms in posterior-basal sections of lungs (34).

Peripheral changes in the lungs are seen as hallmark imaging of COVID-19, and this feature may make it easily accessible by POCUS. The major limitation of lung ultrasound is that it cannot detect lesions that are deep within the lung, as aerated lung blocks transmission of ultrasonography, i.e., the abnormality must extend to the pleural surface to be visible with on ultrasonography examination. Chest CT is required to detect pneumonia that does not extend to the pleural surface.

POCUS has also been shown to be a reliable tool in rapid cardiac evaluation (35). It allows evaluation of pericardial effusion, rough evaluation of ejection fraction and IVC measurements. COVID-19 can affect the heart as well and has shown to cause myocarditis, pericarditis, and congestive heart failure with or without reduced ejection fraction (6). In evaluation of patient with suspected or confirmed COVID-19 POCUS allows evaluation of heart in addition to lungs at bedside. Though pericardial effusion is not common, it has been reported due to COVID-19 and rapid diagnosis can avoid grave complications $(5,36)$. Maintaining fluid balance and avoiding hypervolemia is also essential in management of patients with COVID-19 where POCUS has proven use (37). This added utility is also key in defining role of POCUS in current pandemic. Figure 6 shows side by side comparison of lung collapse/ consolidation, pleural effusion and pericardial effusion in CT scan and POCUS and CT image of pneumothorax.

\section{Consideration for infection transmission risk reduction}

Additional challenge during infectious pandemic is to reduce risk of infection transmission in health care settings while caring for patients. Stringent guidelines including COVID-19 care units, visitor restriction policies, limited 


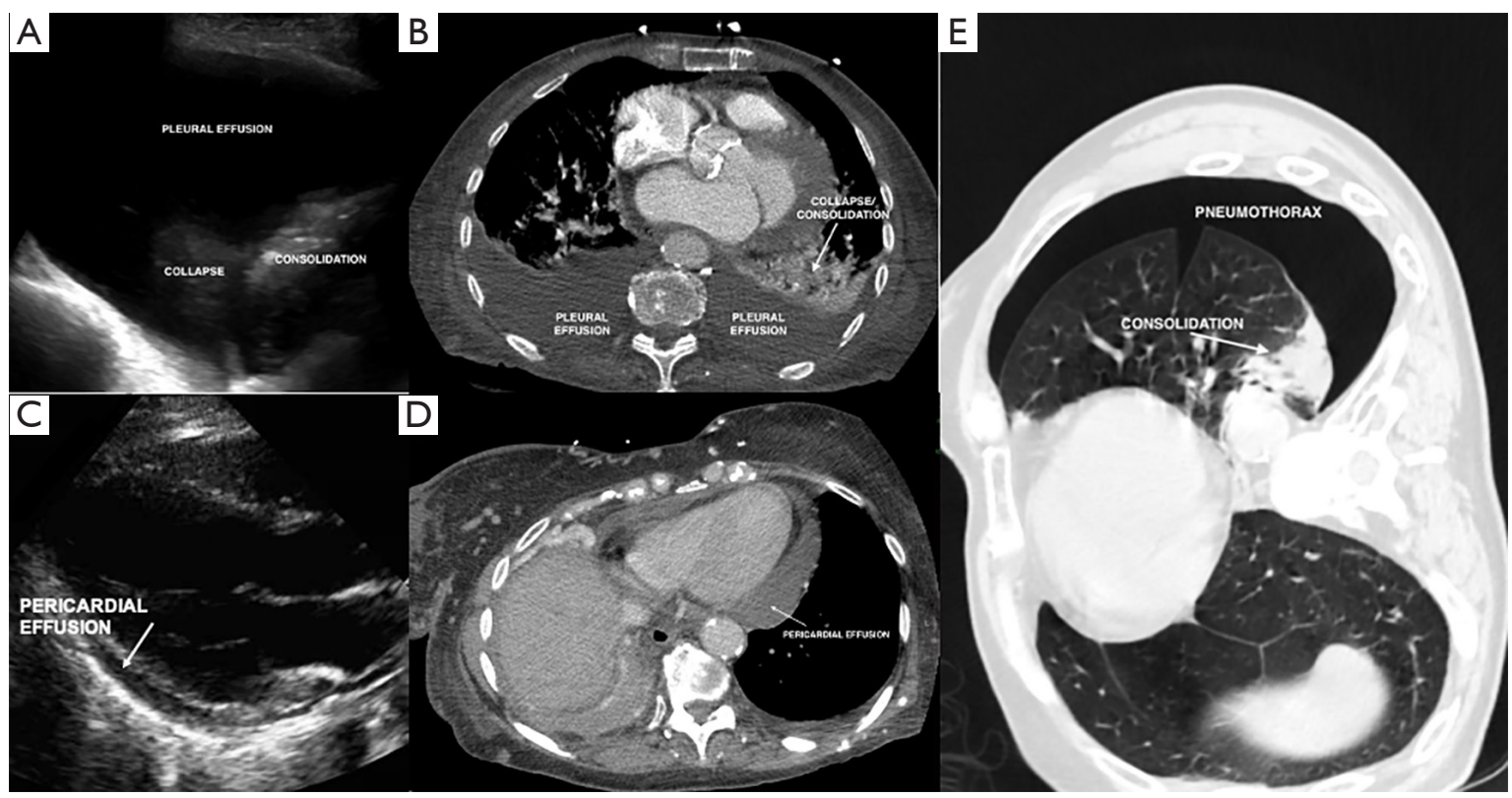

Figure 6 Representative images of lung collapse/consolidation which shows area of "tissue sign" and large anechoic area seen as pleural effusion annotated in (A). Bilateral moderate pleural effusion seen as hypodense fluid collection and consolidation seen as hyperdense area in left lower lobe in axial CT chest with contrast in (B). Similarly, small pericardial effusion seen as anechoic collection in (C) in US and moderate pericardial effusion seen as hypodense fluid collection in (D) in axial CT chest with contrast (white arrows). CT chest lung window shows large pneumothorax in left pleural cavity (annotated) and small area of consolidation in left lower lobe in right lateral decubitus position in (E).

personal stethoscope use, patient care areas and non-patient care areas cleaning protocols are implemented across health care institutes with hope to address this concern. It has been shown that SARS-CoV-2 can survive on surfaces for days (38). Cleaning larger surfaces like CT scan machine after each patient use is complicated. Patient transportation to different areas of hospital for imaging may lead to exposure of additional staff. Localizing care to specific COVID-19 care areas has potential to contain the infection transmission. POCUS can be a useful technology during these situations due to portability.

Disinfection of a portable ultrasound machine can still be challenging and carries risk of transmission of infectious agent from one patient to another. Major bedside ultrasound manufacturer provides COVID-19 resources including cleaning advice of the devices on their website (39). Though risk cannot be completely eliminated disinfection of an ultrasound probe would be significantly easier than disinfection of a CT scanner room. Some basic principles of Ultrasound machine handling and cleaning during COVID-19 pandemic are following (40):

* Place a dedicated machine in the COVID unit 'hot zone';

* Wear gloves when handling machine from room to room;

* Cover US machine with large plastic sheet or part of a gown;

* Cover the entire transducer with plastic sheath or cover non-essential parts with drapes;

* Strip away all ECG leads, gel bottles extra buckets, straps from your machine;

* Clean cables, screen, legs, wheels;

* Use single-use gel packets rather than gel bottles;

* Use a touchscreen device to minimise keyboard and knob handling;

* Wait for up to 3 minutes 'dry time' after using disinfectant wipes before you use the machine again;

* Use a handheld device if available.

Recently ACEP (American College of Emergency Physicians) also published guidelines and cleaning protocol to clean the US machine while and after using on COVID-19 patients, described in a flowchart format in Figure 7 (41).

As this pandemic stretch our imaging and personnel 
Before entering COVID-19 patient's room

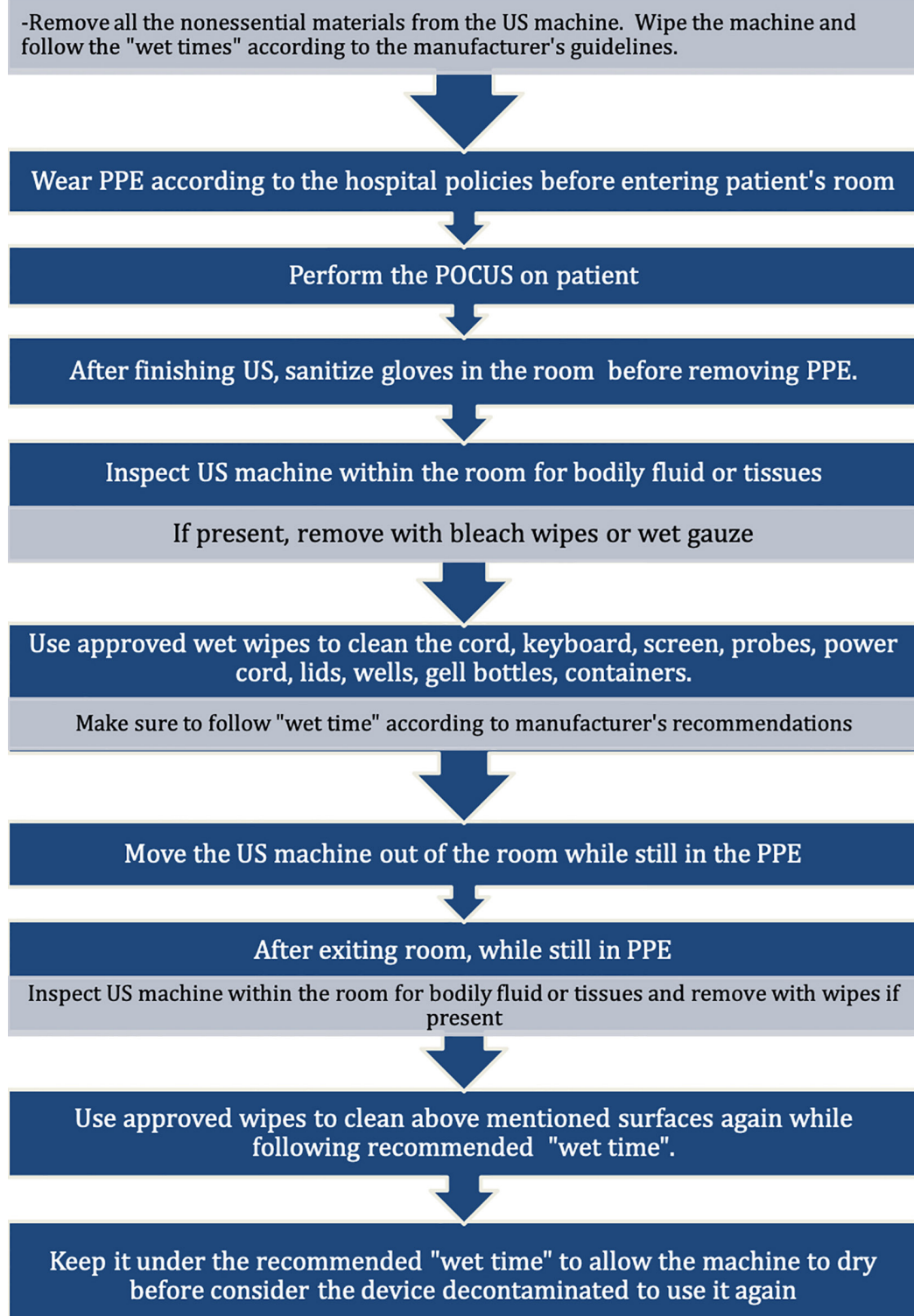

Figure 7 Ultrasound machine cleaning algorithm during POCUS in COVID-19 patient (41).

resources thin and spread to developing part of world including sub-Saharan Africa where CT imaging capabilities are limited, lung ultrasound skills and technology wound be an important weapon in our fight against COVID-19.

\section{Conclusions}

With continuous increase in number of patients in current ongoing pandemic, we need new measures and technology which are quick to scrutinize and triage the patient for further management. Sensitivity of RT-PCR and CT scan imaging findings are high but still questionable due to constant increase in number of affected people. In current pandemic scenario we strongly recommend using of bedside US in patients present with flu like symptoms which is fast, cheap, easy to use and decontaminate. Even though current data on use of POCUS in management of COVID-19 patients are preliminary, further studies would 
be recommended.

\section{Acknowledgments}

Funding: None.

\section{Footnote}

Reporting Checklist: The authors have completed the Narrative Review reporting checklist. Available at http:// dx.doi.org/10.21037/atm-20-3043

Conflicts of Interest: All authors have completed the ICMJE uniform disclosure form (available at http://dx.doi. org/10.21037/atm-20-3043). The authors have no conflicts of interest to declare.

Ethical Statement: The authors are accountable for all aspects of the work in ensuring that questions related to the accuracy or integrity of any part of the work are appropriately investigated and resolved. Verbal consent taken for the use of images of patients. A reasonable individual would be unlikely to object to publication as no personal information has been provided and if required informed consent with documentation can be obtained.

Open Access Statement: This is an Open Access article distributed in accordance with the Creative Commons Attribution-NonCommercial-NoDerivs 4.0 International License (CC BY-NC-ND 4.0), which permits the noncommercial replication and distribution of the article with the strict proviso that no changes or edits are made and the original work is properly cited (including links to both the formal publication through the relevant DOI and the license). See: https://creativecommons.org/licenses/by-nc-nd/4.0/.

\section{References}

1. Available online: https://coronavirus.jhu.edu/map.html

2. Ai T, Yang Z, Hou H, et al. Correlation of Chest CT and RT-PCR Testing for Coronavirus Disease 2019 (COVID-19) in China: A Report of 1014 Cases. Radiology 2020;296:E32-E40.

3. Kanne JP, Little BP, Chung JH, et al. Essentials for Radiologists on COVID-19: An Update-Radiology Scientific Expert Panel. Radiology 2020;296:E113-4.

4. Poggiali E, Dacrema A, Bastoni D, et al. Can Lung US Help Critical Care Clinicians in the Early Diagnosis of
Novel Coronavirus (COVID-19) Pneumonia? Radiology 2020;295:E6.

5. Inciardi RM, Lupi L, Zaccone G, et al. Cardiac Involvement in a Patient With Coronavirus Disease 2019 (COVID-19). JAMA Cardiol 2020. [Epub ahead of print].

6. Kang Y, Chen T, Mui D, et al. Cardiovascular manifestations and treatment considerations in covid-19. Heart 2020. [Epub ahead of print].

7. Zu ZY, Jiang MD, Xu PP, et al. Coronavirus Disease 2019 (COVID-19): A Perspective from China. Radiology 2020;296:E15-E25.

8. Hosseiny M, Kooraki S, Gholamrezanezhad A, et al. Radiology Perspective of Coronavirus Disease 2019 (COVID-19): Lessons From Severe Acute Respiratory Syndrome and Middle East Respiratory Syndrome. AJR Am J Roentgenol 2020;214:1078-82.

9. Ng MY, Lee EY, Yang J, et al. Imaging Profile of the COVID-19 Infection: Radiologic Findings and Literature Review. Radiology 2020. [Epub ahead of print].

10. Zhou S, Wang Y, Zhu T, et al. CT Features of Coronavirus Disease 2019 (COVID-19) Pneumonia in 62 Patients in Wuhan, China. AJR Am J Roentgenol 2020;214:1287-94.

11. Salehi S, Abedi A, Balakrishnan S, et al. Coronavirus Disease 2019 (COVID-19): A Systematic Review of Imaging Findings in 919 Patients. AJR Am J Roentgenol 2020;215:87-93.

12. Liu T, Huang P, Liu H, et al. Spectrum of chest CT findings in a familial cluster of COVID-19 infection. Radiol Cardiothorac Imaging 2020;2:e200025.

13. Yang W, Yan F. Patients with RT-PCR-confirmed COVID-19 and Normal Chest CT. Radiology 2020;295:E3.

14. Wu Y, Xie Y, Wang X. Longitudinal CT Findings in COVID-19 Pneumonia: Case Presenting Organizing Pneumonia. Radiol Cardiothorac Imaging 2020;2:1

15. Pan F, Ye T, Sun P, et al. Time Course of Lung Changes On Chest CT During Recovery From 2019 Novel Coronavirus (COVID-19) Pneumonia. Radiology 2020. [Epub ahead of print].

16. Wang Y, Dong C, Hu Y, et al. Temporal Changes of CT Findings in 90 Patients with COVID-19 Pneumonia: A Longitudinal Study. Radiology 2020;296:E55-E64.

17. Tang L, Zhang X, Wang Y, et al. Severe COVID-19 Pneumonia: Assessing Inflammation Burden with Volumerendered Chest CT. Radiol Cardiothorac Imaging 2020;2:e200044.

18. Bhagra A, Tierney DM, Sekiguchi H, et al. Point-of-Care Ultrasonography for Primary Care Physicians and General 
Internists. Mayo Clin Proc 2016;91:1811-27.

19. Wernecke K, Galanski M, Peters PE, et al. Pneumothorax: evaluation by ultrasound--preliminary results. J Thorac Imaging 1987;2:76-8.

20. Ding W, Shen $Y$, Yang J, et al. Diagnosis of pneumothorax by radiography and ultrasonography: a meta-analysis. Chest 2011;140:859-66.

21. Soldati G, Smargiassi A, Inchingolo R, et al. Is There a Role for Lung Ultrasound During the COVID-19 Pandemic? J Ultrasound Med 2020;39:1459-62.

22. Colmenero M, García-Delgado M, Navarrete I, et al. Utility of the lung ultrasound in the intensive medicine unit. Med Intensiva 2010;34:620-8.

23. Chacko J, Brar G. Bedside ultrasonography: Applications in critical care: Part I. Indian J Crit Care Med 2014;18:301-9.

24. Biswas A, Lascano JE, Mehta HJ, et al. The Utility of the "Shred Sign" in the Diagnosis of Acute Respiratory Distress Syndrome Resulting from Multifocal Pneumonia. Am J Respir Crit Care Med 2017;195:e20-2.

25. Lichtenstein DA, Mezière GA. Relevance of lung ultrasound in the diagnosis of acute respiratory failure: the BLUE protocol. Chest 2008;134:117-25.

26. Vetrugno L, Bove T, Guadagnin GM, et al. Advances in lung ultrasound in critically ill patients. J Emerg Crit Care Med 2019;3:32.

27. Mayo PH, Copetti R, Feller-Kopman D, et al. Thoracic ultrasonography: a narrative review. Intensive Care Med 2019;45:1200-11.

28. Gandhi D, Ojili V, Nepal P, et al. A pictorial review of gall stones and its associated complications. Clin Imaging 2020;60:228-36.

29. Sobh E, Oraby S, Gamal F. Role of thoracic ultrasound in the diagnosis of pneumonia. Eur Respir J 2017;50:PA4113.

30. Long L, Zhao HT, Zhang ZY, et al. Lung ultrasound for the diagnosis of pneumonia in adults: A meta-analysis. Medicine (Baltimore) 2017;96:e5713.

Cite this article as: Gandhi D, Jain N, Khanna K, Li S, Patel L, Gupta N. Current role of imaging in COVID-19 infection with recent recommendations of point of care ultrasound in the contagion: a narrative review. Ann Transl Med 2020;8(17):1094. doi: 10.21037/atm-20-3043
31. Tierney DM, Huelster JS, Overgaard JD, et al. Comparative Performance of Pulmonary Ultrasound, Chest Radiograph, and CT Among Patients With Acute Respiratory Failure. Crit Care Med 2020;48:151-7.

32. Peng QY, Wang XT, Zhang LN; Chinese Critical Care Ultrasound Study Group (CCUSG). Findings of lung ultrasonography of novel corona virus pneumonia during the 2019-2020 epidemic. Intensive Care Med 2020;46:849-50.

33. Huang Yi, Wang S, Liu Y, et al. A Preliminary Study on the Ultrasonic Manifestations of Peripulmonary Lesions of Non-Critical Novel Coronavirus Pneumonia (COVID-19) (February 26, 2020). Available online: https://ssrn. com/abstract=3544750 or http://dx.doi.org/10.2139/ ssrn. 3544750

34. Smith MJ, Hayward SA, Innes SM, et al. Point-of-care lung ultrasound in patients with COVID-19 - a narrative review. Anaesthesia 2020;75:1096-104.

35. Kimura BJ. Point-of-care cardiac ultrasound techniques in the physical examination: better at the bedside. Heart 2017;103:987-94.

36. Allam HH, Kinsara AJ, Tuaima T, et al. Pericardial Fluid in a COVID-19 Patient: Is It Exudate or Transudate? Eur J Case Rep Intern Med 2020;7:001703.

37. Bernier-Jean A, Albert M, Shiloh AL, et al. The Diagnostic and Therapeutic Impact of Point-of-Care Ultrasonography in the Intensive Care Unit. J Intensive Care Med 2017;32:197-203.

38. van Doremalen N, Bushmaker T, Morris DH, et al. Aerosol and Surface Stability of SARS-CoV-2 as Compared with SARS-CoV-1. N Engl J Med 2020;382:1564-7.

39. Available online: https://secure.sonosite.com/covid-19

40. Available online: https://www.stemlynsblog.org/ combatting-covid19-is-lung-ultrasound-an-option/

41. Available online: https://www.acep.org/globalassets/ images/acep-us-machine-cleaning-covid-19.pdf 